\section{PTH-042 ENDOSCOPIC ULTRASOUND (EUS) FOR EVALUATION OF UNEXPLAINED DOUBLE DUCT SIGN WITH NORMAL LIVER BIOCHEMISTRY}

${ }^{1}$ Harry Martin*, ${ }^{2}$ Sreelakshmi Kotha, ${ }^{2}$ kram Hussein, ${ }^{1}$ George Goodchild, ${ }^{1}$ Gavin Johnson, ${ }^{1}$ Steve Pereira, ${ }^{1}$ Michael Chapman, ${ }^{1}$ George Webster, ${ }^{2}$ Sheida Vessal, ${ }^{2}$ Douglas Thorburn, ${ }^{2} J o n a t h a n$ Potts. 'University College London Hospitals NHS Foundation Trust, London, UK ${ }^{2}$ Royal Free London NHS Foundation Trust, London, UK

\subsection{6/gutjnl-2019-BSGAbstracts.67}

Introduction Incidental, unexplained biliary and pancreatitic duct dilatation on imaging performed for other reasons is an increasingly common reason for EUS referral. The radiological 'double duct' (DD) sign is associated with pancreatobiliary (PB) malignancy, particularly in those with abnormal liver function tests (LFTs). However data regarding the yield of pathology at EUS in those with DD and normal live biochemistry are sparse. Our aim was to describe findings at EUS performed for investigation of DD in patients with normal LFTs.

Methods A retrospective review was performed of EUS procedures for investigation of DD across two tertiary HPB referral centres between 2013 and 2018. Those with abnormal or unknown liver biochemistry, or where expert review of crosssectional imaging had indicated a mass lesion, stricture or other identifiable cause were excluded.

Results 46 patients were included, median age 64 years, 78\% female/22\% male. Long-term opiate use was documented in $17(37 \%)$.

Positive findings were identified at EUS in 10 (21.7\%); chronic pancreatitis $(n=5)$, side branch intra-ductal papillary mucinous neoplasm (IPMN) $(n=2)$, benign papillary fibrosis $(n=2)$ and choledocholithiasis $(n=1)$. Ductal dilatation was not seen at EUS in $3(6.5 \%)$ and DD was present with no cause identified in 33 (71.7\%), of whom 14 (30.4\%) had documented opiate use. Both cases of IPMN lacked high-risk features and the patients remained well with a minimum of 18 months surveillance.
One endoscopic complication occurred in a patient without cause for DD sign identified at EUS, who sustained an endoscopic perforation requiring hepatico-jejunostomy.

Two cases of ampullary carcinoma were noted during case identification in patients with minimally elevated LFTs undergoing EUS for DD.

Conclusions In this cohort with DD and normal liver biochemistry there were no EUS-diagnosed malignancy and the yield of benign pathology was $\sim 20 \%$, similar to that previously reported ${ }^{4}$. However, this should be interpreted with caution given the 2 cases of malignancy, not included in this cohort, found with only mild LFT abnormalities and further prospective studies are needed.

\section{REFERENCES}

1. Krishna $\mathrm{N}$, et al. Dilation of both pancreatic duct and the common bile duct on computed tomography and magnetic resonance imaging scans in patients with or without obstructive jaundice. Pancreas 2012;41:767-72.

2. Kofi W Oppong, et al. Endoscopic ultrasound in patients with normal liver blood tests and unexplained dilatation of common bile duct and or pancreatic duct. Scandinavian Journal of Gastroenterology 2014;49(4):473-480.

\section{PTH-043 THE ACCURACY OF ULTRATHIN ENDOSCOPY IN THE DIAGNOSIS OF BARRETT'S OESOPHAGUS: SYSTEMATIC REVIEW AND META-ANALYSIS}

1John McGoran*, ${ }^{2} J a c o b o$ Ortiz Fernández-Sordo, ${ }^{1} J$ John SDe Caestecker, ${ }^{3}$ Rehan Haidry, ${ }^{3}$ Laurence B Lovat, ${ }^{2}$ Krish Ragunath, ${ }^{3}$ Sarmed S Sami. ' University Hospitals Of Leicester, Leicester, UK; ${ }^{2}$ Nottingham University Hospitals NHS Trust, Nottingham, UK; ${ }^{3}$ University College London, London, UK

\subsection{6/gutjnl-2019-BSGAbstracts.68}

Introduction Screening for Barrett's oesophagus (BO) may be considered for those at high risk. Unsedated Ultrathin Endoscopy (UE) has been proposed as a more acceptable, cost-effective alternative to conventional oesophagogastroduodenoscopy (C-OGD) but individual studies on the diagnostic performance of UE are often underpowered. The pooled diagnostic

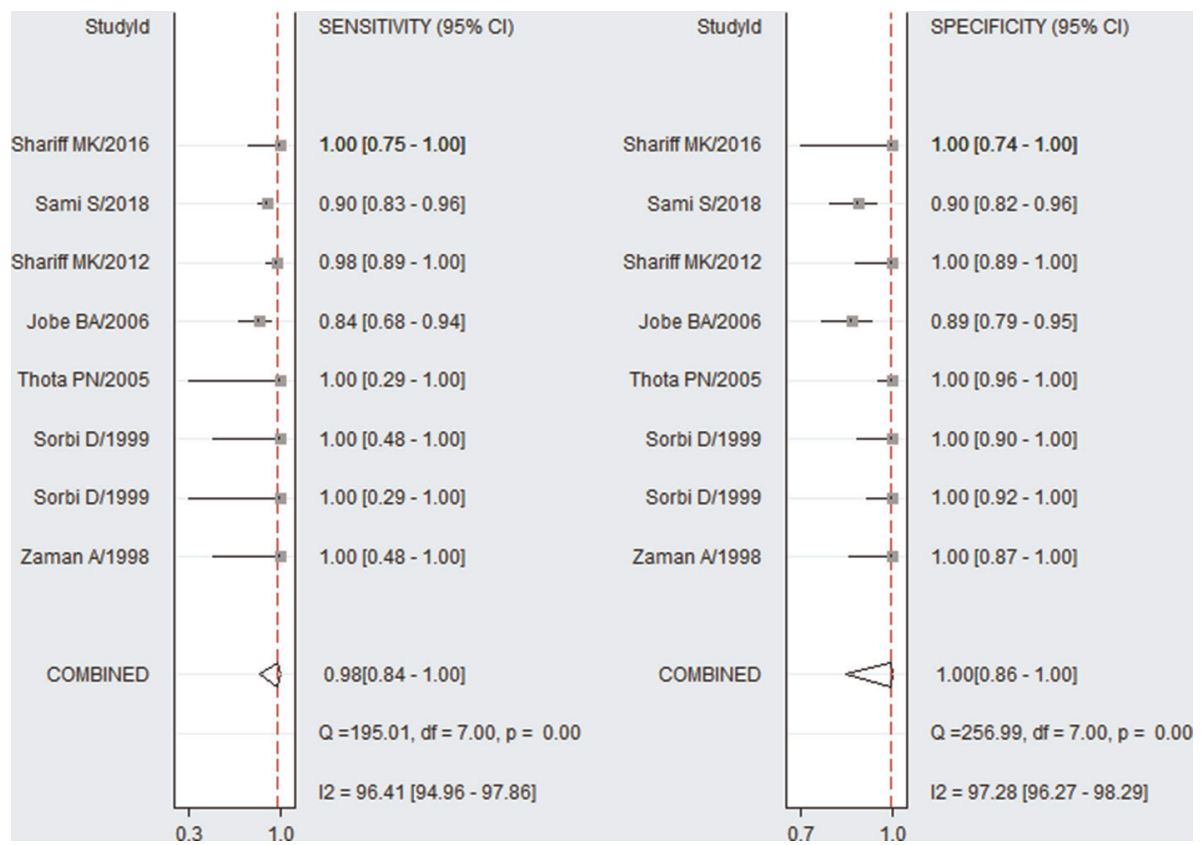

Abstract PTH-043 Box 1 Forest plot of trials including in meta-analysis. 